\title{
Efficient Unilateral Authentication Mechanism for MIPv6
}

\author{
Yoon-Su Jeong ${ }^{1}$, Bong-Keun Lee ${ }^{2}$, Keon-Myung Lee ${ }^{1}$, and Sang-Ho Lee ${ }^{1}$ \\ 1 Department of Computer Science, Chungbuk National University, Chungju, \\ Chungbuk, Korea \\ bukmunro@netsec.cbnu.ac.kr, \{kmlee, shlee\}@chungbuk.ac.kr \\ 2 Department of Multimedia Computer, Busan Kyungsang College, \\ Chungbuk, Korea \\ rbk@bsks.ac.kr
}

\begin{abstract}
We present a unilateral authentication protocol for protecting IPv6 networks against abuse of mobile IPv6 primitives. The proposed protocol imposes minimal computational requirements on mobile nodes, uses as few messages as possible. It is also easy to implement, economic to deploy and lightweight in use. We formally verifies the correctness of the protocol using the finite-state analysis tool mur $\phi$, which has been used previously to analyze hardware designs and security properties of several protocols.
\end{abstract}

\section{Introduction}

Recently mobility technology is regarded as indispensable feature in internet area according to widely use of mobile nodes such as notebook computer, internet accessible mobile phone. Mobile IPv6 is a key protocol that supports mobility in IPv6 network. But Mobile IPv6 specification does not specify how to gain access to network when mobile node is away from its home.

The most difficult adoption issue for any form of authenticated Mobile IPv6 is reliance on authentication infrastructure. Recent Diameter specification deals with IPv6 mobility support [1]. However, The [1] does not state any specific protocol to be used between mobile node and AAA client. This paper proposes efficient user authentication to provide a minimum level of unilateral authentication of binding update. We assume that MIPv6 is used to authenticate the user and Diameter conveys MIPv6 authentication message between foreign AAA(AAAF) and home $\mathrm{AAA}(\mathrm{AAAH})$. In this paper, diameter is responsible for supporting IP mobility features such as home agent assignment and key distribution which is needed to securely send BU message to its home agent.

\section{Related Works}

Brandner, Mankin and Schiller [4] proposed a framework called Purpose Built Keys. An advantage of this framework is that it does not require any security infrastructure. However, purpose-built keys provide authentication if and only 
if the initial hash of the public key is received correctly by correspondent node (CN). This might not be the case. An attacker could intercept the hash and send the hash of a different key (which it owns) to CN. Subsequently, it can pretend to be mobile node (MN) without $\mathrm{CN}$ being any wiser. The authors of the draft were, of course, aware of this weakness.

Le and Faccin[2] proposed two protocols for authenticating binding updates. The first assumes that both the MN and the CN share security associations with two AAA servers. The second protocol proposed in [2] involves an unauthenticated Diffie-Hellman key exchange between MN and CN. The resulting key is subsequently used to authenticate binding updates. The authors recognize that this protocol is vulnerable to a man-in-the-middle(MITM) attack but state that "due to the properties of IP" such an attack will always be detected.

\section{Proposed Protocol}

In this section, our main goal is to institute a mechanism for setting up security associations between $\mathrm{MN}-\mathrm{CN}$ pairs that will allow binding updates to be authenticated.

\subsection{Assumption}

We consider the following design assumptions:

- Each MN(Mobile Node) has a pre-established bidirectional security association with its HA(Home Agent) using which they can authenticate each other.

- HA's are capable of authenticating each other using public key cryptography.

- Identity verification should not rely on the existence of a global PKI.

- Minimize the number of messages and bytes sent between the participating entities.

- Consider the computational capabilities of the MN's and CN's.

- Resist to DoS attacks that CN does not need to create state before the third message.

\subsection{Protocol Description}

Our protocol considers (a) an initialization phase in which $\mathrm{MN}$ and $\mathrm{CN}$ set up an authentication key and (b) an update phase in which MN sends an authenticated binding update to $\mathrm{CN}$ using the key obtained from phase (a).

Initialization Phase. The initialization phase for the protocols are the elliptic curve parameters that are common to both entities and consist of an elliptic curve E defined over a finite field $F_{q}$, generating element $\mathrm{G}$ (a point of the elliptic curve $) \in \mathrm{E}\left(F_{q}\right)$, $\mathrm{n}$ is order of $\mathrm{G}$ in $\mathrm{E}\left(F_{q}\right)$, and $\mathrm{h}$ is cofactor of n,i.e.,h= $\sharp \mathrm{E}\left(F_{q}\right) / \mathrm{n}$.

We will assume that static public keys are exchanged via certificates. $\operatorname{Cert}_{H A_{M N}}$ denotes $H A_{M N}$ 's public-key certificate, containing her static public key $Y_{a}$, 


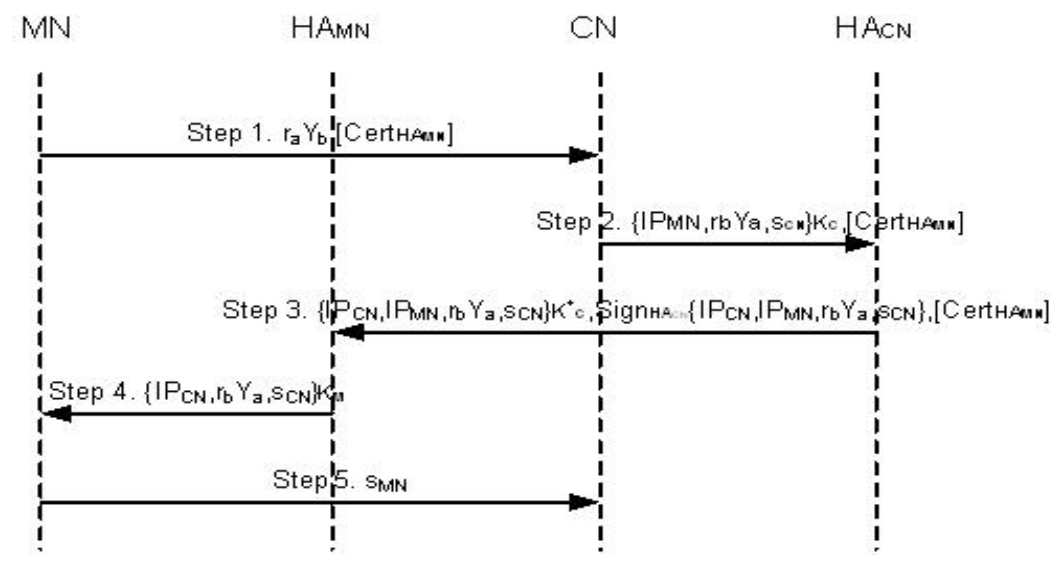

Fig. 1. Registration protocol

ephemeral key(random number) $r_{a}$ and a certifying authority $H A_{C N}$ 's signature. The security of proposed protocol in this paper is based on the Diffie-Hellman problem in elliptic curve group(ECDHP). The protocol is shown in Figure 1 as follows.

- Step 1: A MN send a message $\left(r_{a} Y_{b}\right.$ and $\left.\operatorname{Cert}_{H A_{M N}}\right)$ to $\mathrm{CN}$ when MN already knows a group and generator that is acceptable to $\mathrm{CN}$.

- Step 2: Upon receiving message 1, CN generates $r_{b} Y_{a}$, computes $K_{D}==$ $\mathrm{h}\left(\left(r_{b} / x_{b}\right) M_{a}+x_{b} Y_{a}\right)=\mathrm{h}\left(r_{a} r_{b}+x_{a} x_{b}\right) \mathrm{G}$ and $S_{C N}$ and sends a message to $H A_{C N}$ encrypted with their shared key, $K_{C}$.

- Step 3: $H A_{C N}$ forwards to $H A_{M N}$ the session parameters that it recovers by decrypting message 2 . It is necessary to include CN's IP address since the same MN might be executing this protocol parallely with multiple correspondent nodes.

- Step 4: $H A_{M N}$ forwards the session parameters to MN in message 4, encrypted with $K_{M}$, their shared key.

- Step 5: MN encrypts the session parameters with the newly computed elliptic curve secret based on the ECDLP.

In this protocol, $\mathrm{CN}$ authenticates herself to MN through the chain of trust $\mathrm{CN} \rightarrow H A_{C N} \rightarrow H A_{M N} \rightarrow \mathrm{MN}$. MN could also use the same trust chain in the other direction to authenticate herself to $\mathrm{CN}$.

Update Phase. Once $\mathrm{MN}$ and $\mathrm{CN}$ have set up a shared secret, $K_{D}, \mathrm{MN}$ can easily send an authenticated binding update(BU) by executing the following Step 1 protocol.

Identity, BU, $\mathrm{h}\left(K_{D}\right.$, Identity, BU)

Here, $\mathrm{h}(\ldots)$ is a keyed cryptographic hash function [3]. 


\section{Analysis of the Protocol}

The use of elliptic curve key exchange ensures that the protocol provides perfect forward secrecy(PFS)[5]. If the perfect forward secrecy is not a requirement, an alternative would be to replace the exchange of certificate by an exchange of nonces $\left(n_{M N}\right.$ and $\left.n_{C N}\right)$. $n_{M N}$ could be sent in the clear in Step 1, while $n_{C N}$ is sent encrypted in Step 2, 3, 4. The shared secret could be derived from a hash of these nonces, e.g., $\operatorname{HMAC}\left(n_{M N}, n_{C N}\right)$.

A key exchange protocol requires each participant to use some fresh information in every run of the protocol. Any adversary which possesses a certificate could intercept Step 1 and then send Step 3 without $H A_{M N}$ being any wiser.

The basic 5-Step protocol is susceptible to denial of service attacks. A useful property of our protocol is that since home agents do not create state, memory denial of service attacks are not possible on the home agents. Preventing computation denial of service attacks on the home agents reduces to the problem of detecting, without performing expensive computations, whether a message has been replayed. Preventing denial of service attacks on $H A_{M N}$ without adding extra message, appears to be more difficult. Adding a sequence number to message 3 and including it in the signature alleviates the problem somewhat.

\section{Conclusions}

In response to the requirement that all location information about a mobile node in IPv6 should be authenticated, we proposed a protocol for authenticating binding updates. The other requirements are taken into accounts as well: the computational load on the nodes and on the routers is minimized, by eliminating expensive encryption operations and keeping the number of steps at minimum. Therefore, we believe that our protocol addresses the main issues in Mobile IPv6 authentication and makes best use of whatever infrastructure is available.

\section{References}

[1] A. J. Menezes, P. C. van Oorschot, S. A. Vanstone. Handbook of Applied Cryptography. CRC Press, (1996).

[2] F. Le, S. M. Faccin. Dynamic Diffie Helman based Key Distribution for Mobile IPv6. Internet Draft, (April 2001).

[3] S. Bradner, A. Mankin, J. I. Schiller. A framework for purpose Built Keys(PBK). Internet Draft. (February 2001).

[4] H. Krawczyk, M. Bellare, R. Canetti. HMAC:Keyed-Hashing for Mesage Authentication. RFC 2104. (February 1997).

[5] D. Dill. The Mur $\phi$ Verification System. In Proc. 8th International Conference on Computer Aided Verification, pages 390-393. (1996). 